\title{
Prevalencia e incidencia de hiperpotasemia en población española con insuficiencia cardiaca crónica: revisión sistemática y relevancia poblacional
}

Álvaro Hernáez ${ }^{\mathrm{a}, \mathrm{b}}$, Juan F. Delgado ${ }^{\mathrm{c}, \mathrm{d}}$, Juan Cinca ${ }^{\mathrm{d}, \mathrm{e}}$, Francisco Fernández-Avilés ${ }^{\mathrm{d}, \mathrm{f}}$, Jaume Marrugat ${ }^{\mathrm{d}, \mathrm{g}, *}$

a. Grupo de investigación en Riesgo Cardiovascular y Nutrición-REGICOR, Institut Hospital d'Investigacions Mèdiques (IMIM), Barcelona, España.

b. CIBER de Fisiopatología de la Obesidad y Nutrición (CIBEROBN), Instituto de Salud Carlos III, Madrid, España.

c. Fundación Investigación i+12, Servicio de Cardiología, Hospital Universitario 12 de Octubre. Facultad de Medicina, Universidad Complutense de Madrid, Madrid.

d. CIBER de Enfermedades Cardiovasculares (CIBERCV), Instituto de Salud Carlos III, Madrid, España.

e. Servicio de Cardiología, Hospital de la Santa Creu i Sant Pau, Universitat Autònoma de Barcelona, Barcelona, España

f. Departamento de Cardiología, Instituto de Investigación Sanitaria Hospital General Universitario Gregorio Marañón, Madrid, España

g. Grupo de investigación en Epidemiología y Genética CardiovascularREGICOR, Institut Hospital d'Investigacions Mèdiques (IMIM), Barcelona, España.

\section{Autor de correspondencia}

Jaume Marrugat, MD, PhD

Institut Hospital del Mar d'Investigacions Mèdiques (IMIM)

Carrer Doctor Aiguader 88, 08003, Barcelona, España 
Teléfono: (+34) 933160733

Fax: (+34) 933160796

e-mail: imarrugat@imim.es 


\section{RESUMEN ESTRUCTURADO}

Antecedentes y objetivo. La hiperpotasemia $\left(K^{+} \geq 5,5 \mathrm{mmol} / \mathrm{L}\right)$ es un desequilibrio iónico grave cuando aparece en pacientes con insuficiencia cardiaca crónica (ICC) ya que incrementa el riesgo de fibrilación ventricular. No existen estimaciones del número de pacientes que la sufren y que la desarrollan cada año en España ni en otro país de nuestro entorno. Nuestro objetivo estimar la prevalencia y la incidencia de hiperpotasemia en pacientes con ICC en España.

Materiales y métodos. A partir de una búsqueda bibliográfica sistemática, calculamos la prevalencia de ICC moderada/grave (fracción de eyección sistólica <40\%) en población europea/norteamericana en un meta-análisis. A partir de otra búsqueda bibliográfica sistemática, calculamos la prevalencia de hiperpotasemia en individuos con ICC en otro meta-análisis y estimamos su incidencia anual. Considerando los anteriores valores y la pirámide de población española en 2016, estimamos el número de individuos con ICC que presentan actualmente o que desarrollan cada año hiperpotasemia en España.

Resultados. Estimamos que unos 17.100 individuos (unos 10.000 hombres y 7.100 mujeres) de los 508.000 pacientes con ICC presentan hiperpotasemia en España. Asimismo, estimamos que unos 14.900 pacientes con ICC (unos 9.500 hombres y 5.400 mujeres) la desarrollan cada año.

Conclusiones. Aproximadamente 1 de cada 30 pacientes con ICC presentan niveles plasmáticos de potasio por encima de $5,5 \mathrm{mmol} / \mathrm{L}$. Así, parece necesario el estudio de nuevos agentes que corrijan la hiperpotasemia mediante los ensayos clínicos pertinentes, con el fin de incorporarlos a la práctica clínica con todas las garantías.

PALABRAS CLAVE: hiperpotasemia; potasio; insuficiencia cardiaca crónica; prevalencia; incidencia; estimación. 


\section{ABSTRACT}

Background and objective. Hyperkalemia $\left(K^{+} \geq 5.5 \mathrm{mmol} / \mathrm{L}\right)$ is a severe ionic imbalance in patients with chronic heart failure (CHF) since it increases the risk of suffering ventricular fibrillation. There is no estimation of the number of patients that present or develop it every year neither in Spain nor in other similar countries. Our objective was to estimate the prevalence and incidence of hyperkalemia in $\mathrm{CHF}$ patients in Spain.

Materials and methods. From a systematic search, we calculated the prevalence of moderate/severe CHF (systolic ejection fraction $<40 \%$ ) in European/North American population in a meta-analysis. From another systematic search, we calculated the prevalence of hyperkalemia in CHF patients in another meta-analysis and estimated its annual incidence. Considering the previous values and Spanish population pyramid in 2016, we estimated the number of CHF patients that present nowadays or that develop every year hyperkalemia in Spain.

Results. We estimated that around 17,100 subjects (10,000 men and 7,100 women) of the 508,000 CHF patients in Spain present hyperkalemia. Likewise, around 14,900 CHF patients develop it every year (9,500 men and 5,400 women).

Conclusions. Approximately 1 in $30 \mathrm{CHF}$ patients presents potassium levels above $5.5 \mathrm{mmol} / \mathrm{L}$. Thus, the study of novel anti-hyperkalemia agents in clinical trials seems necessary, in order to incorporate these tools to clinical practice with all the guarantees.

KEYWORDS: hyperkalemia; potassium; chronic heart failure; prevalence; incidence; estimation. 


\section{'INTRODUCCIÓN}

El potasio $\left(\mathrm{K}^{+}\right)$es un mineral esencial para numerosas funciones fisiológicas, entre las que destaca el mantenimiento del potencial de membrana de las células. Las alteraciones por exceso en los niveles plasmáticos de potasio (hiperpotasemia, definida generalmente como niveles de $\mathrm{K}^{+} \geq 5,5 \mathrm{mmol} / \mathrm{L}$ ) se asocian a trastornos del potencial de acción celular en los que el potasio es clave, como son la conducción y excitabilidad cardiaca ${ }^{1}$. Niveles excesivos de potasio se asocian con una mayor mortalidad total e intra-hospitalaria ${ }^{2-4}$, por las numerosas alteraciones en el ritmo y la contracción del miocardio con las que se $\operatorname{asocian}^{5}$ (que pueden conducir a la aparición de fibrilación ventricular y asistolia) $)^{1,6}$.

Los pacientes con insuficiencia cardiaca crónica (ICC) deben conseguir un control estricto de sus niveles de potasio ya que el sustrato de su enfermedad agrava las posibles consecuencias de la hiperpotasemia y viceversa ${ }^{6,7}$. Estos pacientes suelen presentar otras comorbilidades que favorecen el agravamiento de la hiperpotasemia (insuficiencia renal, diabetes mellitus tipo 2). Además, pueden estar tratados con fármacos que contribuyen a aumentar los niveles plasmáticos de potasio (inhibidores del enzima convertidora de angiotensina -IECAs-, antagonistas del receptor de angiotensina-II -ARA-II- o antagonistas del receptor de mineralocorticoides -ARMs)$^{6,8}$. Por otro lado, el desarrollo de hiperpotasemia impide que se alcancen las dosis terapéuticas en el $4 \%$ de los pacientes tratados con IECAs, el $2 \%$ de los tratados con ARA-Il y el 8\% de los tratados con ARMs en España, así como es responsable del 7\% de las contraindicaciones de IECAs y ARA-II y el 31\% de las de ARMs en nuestro país ${ }^{9}$.

A pesar de todo ello, no se conoce el número exacto de pacientes con ICC que presentan o desarrollan cada año hiperpotasemia en España ni en países de nuestro

\footnotetext{
${ }^{1}$ ARA-II: antagonista del receptor de angiotensina-II; ARM: antagonista del receptor de mineralocorticoides; FEVI: fracción de eyección del ventrículo izquierdo; ICC: Insuficiencia cardiaca crónica; IECA: Inhibidor de la enzima convertidora de angiotensina.
} 
entorno, como paso previo a la discusión de si es pertinente desarrollar nuevos abordajes terapéuticos para controlar esta alteración iónica y sus consecuencias. Así, el objetivo de este trabajo es estimar el número de pacientes con ICC que presentan hiperpotasemia en la actualidad y que la desarrollan anualmente en la población española.

\section{MÉTODOS}

\section{Estimación de ICC en población caucásica}

Para calcular el número de casos de hiperpotasemia en pacientes con ICC en España, en primer lugar es necesario determinar el número de pacientes que sufren ICC en nuestro país. A pesar de que el estudio PRICE describió los valores de prevalencia de ICC en población española ${ }^{10}$, dichos valores se encontraban muy por encima de los descritos en otros trabajos realizados en población europea y norteamericana, lo que se ha atribuido a particularidades técnicas de dicho estudio y no tanto a que las tasas de ICC sean mayores en España ${ }^{11}$. Para calcular un valor que reflejase de forma precisa la prevalencia de ICC en poblaciones como la española, realizamos una búsqueda bibliográfica en PubMed de todos aquellos estudios realizados en población general europea y norteamericana en los que se describiese la prevalencia de ICC moderada-grave con fracción de eyección del ventrículo izquierdo (FEVI) deprimida $(\mathrm{FEVI} \leq 40 \%)^{12}$ distribuida por sexo y grupos de edad. Para ello, buscamos artículos que contuvieran el término "insuficiencia cardiaca" en el título; "prevalencia" o "carga" en el título o el resumen; "fracción de eyección", "sístole" o "sistólica" en el título o el resumen; y "población general” o "sano" en el título o el resumen (fecha de búsqueda 01/09/2017). Dos de los autores del trabajo (A.H. y J.M.) revisaron por separado los títulos y resúmenes del total de referencias obtenidas $(N=91)$ y los clasificaron como "relevantes", "de relevancia posible" o "irrelevantes". Aquellos artículos que se 
clasificaron como "relevantes" o "de relevancia posible" por ambos autores fueron revisados en su totalidad ( $N=22)$, resolviéndose las discrepancias por consenso. De esta última selección, se conservaron los datos de aquellas publicaciones que recogiesen las tasas de prevalencia de ICC con una FEVI $\leq 40 \%$, en población europea o norteamericana y que proporcionasen el número exacto de casos de ICC en cada grupo de edad y sexo $(N=6)^{13-17}$. El estudio PRICE no fue finalmente incluido en el meta-análisis por aumentar significativamente la heterogeneidad de los datos. A partir de estos datos, se estimaron las prevalencias de ICC en hombres y mujeres de $45-54,55-64,65-74$ y 75 o más años, mediante distintos meta-análisis para cada franja de edad y sexo. Cuando alguno de los estudios describiese la prevalencia en franjas de edad distintas a las anteriores (p.ej., por décadas -50-59 años-), la mitad de los casos y del número total de personas de dicho grupo se asignaron a la franja inferior (45-54 años) y la otra mitad a la franja superior (55-64 años). Cuando la heterogeneidad de los datos no fuese estadísticamente significativa, estos se metaanalizaron mediante modelos de efectos fijos, mientras que si la heterogeneidad entre los datos resultó estadísticamente significativa, estos se meta-analizaron mediante modelos de efectos aleatorios. Los anteriores análisis se realizaron mediante el paquete "meta"18 en R Software, versión 3.4.1 ( $R$ : A language and environment for statistical computing. R Foundation for Statistical Computing. Vienna, Austria). Aplicando estas tasas de prevalencia a la pirámide demográfica española en $2016^{19}$ se calculó el número de hombres y mujeres con ICC en las cuatro franjas de edad de interés.

\section{Estimación de la prevalencia y la incidencia de hiperpotasemia en ICC}

Se realizó otra búsqueda bibliográfica sistemática en PubMed para identificar aquellos estudios de pacientes con ICC en los que se determinasen los niveles de potasio o la presencia de hiperpotasemia. Para ello, se buscaron los artículos que contuvieran "insuficiencia cardiaca" en el título; los conceptos "cohorte", "prospectivo", 
"seguimiento", "transversal" o "registro" en el título o el resumen del artículo; y los conceptos "potasio" o "hiperpotasemia" en el título o en el resumen del trabajo (fecha de búsqueda: 01/09/2017). Tres de los autores del trabajo (A.H., J.F.D. y J.M.) revisaron por separado todos los títulos y resúmenes del total de referencias obtenidas $(N=157)$ y los calificaron como "relevante", "de relevancia posible" o "irrelevante". Aquellos trabajos que se clasificaron como "relevantes" o "de relevancia posible" por los tres autores fueron revisados en su totalidad $(N=21)$, resolviendo las discrepancias por consenso. De esta última selección, dos estudios presentaron valores de prevalencia de hiperpotasemia en cohortes de $\mathrm{ICC}^{20,21}$, mientras que uno reportó valores de incidencia anual ${ }^{22}$.

La prevalencia de hiperpotasemia se estimó de nuevo en un meta-análisis obteniendo la proporción de individuos con $\mathrm{K}^{+} \geq 5,5 \mathrm{mmol} / \mathrm{L}$ en el total de sujetos con ICC en los dos estudios. El meta-análisis volvió a realizarse de acuerdo con la metodología descrita en el anterior apartado, en el paquete "meta"18 en R Software, versión 3.4.1 ( $R$ : A language and environment for statistical computing. R Foundation for Statistical Computing. Vienna, Austria). La incidencia de nuevos casos de hiperpotasemia por sexo (por 100 años-persona) se calculó a partir de las tasas de incidencia por franjas de edad, ponderándolas en función de la proporción de casos aparecidos en el grupo de hombres $(57,9 \%)$ y mujeres $(42,1 \%)$ observadas en la única publicación seleccionada $^{22}$. Con estos datos, se estimó la incidencia de hiperpotasemia en hombres y mujeres con ICC de 45-54, 55-64, 65-74 y 75 o más años. Finalmente, aplicamos los anteriores valores de prevalencia e incidencia de hiperpotasemia al número de pacientes con ICC en España para calcular los sujetos que la presentan en la actualidad o la desarrollan anualmente.

\section{RESULTADOS}




\section{Estimación de la prevalencia de ICC en poblaciones europeas/norteamericanas}

Nuestros meta-análisis indicaron una prevalencia estimada de ICC del 3,68\% en hombres y del 1,66\% en mujeres en poblaciones europeas/norteamericanas, con progresión ascendente a medida que aumenta la edad: en hombres, un 0,58\% de 45 54 años, un 2,17\% de 55-64 años, un 4,48\% de 65-74 años y un 7,20\% con 75 o más años; en mujeres, un 0,09\% de 45-54 años, un 0,52\% de 55-64 años, un 1,78\% de 6574 años y un $5,78 \%$ con 75 o más años (Figuras 1 y 2).

\section{Prevalencia de hiperpotasemia en ICC en España}

La prevalencia de hiperpotasemia en ICC estimada en nuestro meta-análisis fue del 3,37\% (Figura 3). Este dato nos permitió estimar que 9.969 hombres y 7.162 mujeres presentaron hiperpotasemia e ICC en España en 2016 (Tabla 1).

\section{Incidencia de hiperpotasemia en ICC en España}

Ponderando las tasas de incidencia en función de la proporción de los casos totales acontecidos en hombres y mujeres, estimamos una incidencia de 1,97, 2,21, 3,16, 3,58 y 3,62 casos/100 años-persona para hombres y de $1,43,1,61,2,30,2,60$ y 2,63 casos/100 años-persona para mujeres de 20-49, 50-59, 60-69, 70-79 y 80 o más años, respectivamente. Los anteriores datos nos permitieron estimar que 9.553 hombres y 5.356 mujeres con ICC desarrollaron hiperpotasemia en 2016 (Tabla 2).

\section{DISCUSIÓN}

En el presente estudio, a partir de una revisión bibliográfica sistemática y el uso de información demográfica actualizada, hemos estimado el número de pacientes con ICC moderada o grave con $\mathrm{FEVI} \leq 40 \%$ que presentan hiperpotasemia ( $\mathrm{K}^{+}$plasmático $\geq 5,5 \mathrm{mmol} / \mathrm{L}$ ) o que la desarrollan anualmente en España. Según nuestras 
estimaciones, de los aproximadamente 508.000 pacientes con ICC moderada o grave actualmente en nuestro país, aproximadamente 17.100 pacientes presentan hiperpotasemia (unos 10.000 hombres y unas 7.100 mujeres). Asimismo, se producen alrededor de 14.900 nuevos casos anuales (unos 9.500 hombres y unas 5.400 mujeres). Hasta donde sabemos, esta es la primera vez en la que se ha realizado una estimación poblacional del número de personas con ICC que presentan o que desarrollarán hiperpotasemia anualmente en España u otro país de características similares.

La hiperpotasemia es un desequilibrio iónico infradiagnosticado, generalmente mal controlado en los pacientes con ICC y una causa frecuente de retirada de fármacos que son eficaces en la reducción de morbimortalidad de los pacientes con IC y FEVI deprimida $^{9,20,11}$. En este sentido, el presente estudio nos ha permitido estimar que unos 17.100 pacientes de este colectivo ( 10.000 hombres y 7.100 mujeres) presentan niveles elevados de potasio en la actualidad, y que aproximadamente 14.900 pacientes la desarrollan cada año (9.500 hombres y 5.400 mujeres). Todos estos pacientes serían tributarios de un tratamiento específico. Las cifras de prevalencia e incidencia y la problemática que la hiperpotasemia plantea en el tratamiento de la IC con FEVI deprimida justifican el estudio de nuevas estrategias terapéuticas antihiperpotasémicas seguras tales como los nuevos fármacos quelantes del potasio a nivel de tracto gastrointestinal ZS-9 o patiromer ${ }^{23,24}$, considerando que los agentes disponibles actualmente son mal tolerados ${ }^{1}$. La realización de estudios de fase III para nuevos medicamentos es posible si se encuentran entre 300 y 3.000 pacientes que podrían beneficiarse de dichos tratamientos ${ }^{25}$.

Las cifras de prevalencia de hiperpotasemia en nuestra estimación son ligeramente más elevadas que las de incidencia anual: existiendo un total de unos 17.000 casos prevalentes de pacientes con ICC moderada/grave e hiperpotasemia, se diagnostican unos 15.000 nuevos cada año. Que la prevalencia de hiperpotasemia sea sólo ligeramente superior a la incidencia podría explicarse considerando que la mayoría de 
los nuevos casos de hiperpotasemia se corregirían o revertirían al poco tiempo (por ejemplo, mediante la retirada de los medicamentos responsables de su inducción ${ }^{9}$ ), mientras que sólo los casos más graves persistirían y formarían parte del grupo de pacientes hiperpotasémicos crónicos o recurrentes (aquellos pacientes en los que la retirada o la reducción de la dosis de IECAs, ARA-II o ARMs no es asumible o en los que el deterioro de la función renal es más marcado). Todo ello vuelve a poner de manifiesto el interés de investigar nuevas herramientas terapéuticas para tratar estos "casos resistentes" y reducir la prevalencia de hiperpotasemia de forma segura. La principal fortaleza de nuestro estudio es que se trata de la primera estimación del número de personas con ICC que presentan o que desarrollarán hiperpotasemia anualmente a partir de datos de estudios epidemiológicos recientes y estimaciones demográficas. Sin embargo, también presenta limitaciones. En primer lugar, nuestros cálculos se han realizado en base a datos de pocos estudios (dos para el caso de la prevalencia de hiperpotasemia, y uno para la incidencia). Sería conveniente repetir este tipo de análisis más adelante, para incluir datos de nuevos trabajos. En segundo lugar, los anteriores cálculos se han realizado extrapolando resultados de otras poblaciones, lo que podría comprometer la validez externa de algunas de las conclusiones del trabajo. En tercer lugar, la prevalencia de hiperpotasemia en ICC se expresó como una cifra única, no estratificada por grupos de edad y sexo debido a la ausencia de suficientes datos publicados en dichos estratos. Aunque varios de los estudios examinados han presentado resultados parciales en algunos de estos grupos, se constata que la edad y el sexo tienen escaso efecto sobre la prevalencia de hiperpotasemia en $\operatorname{ICC}^{21,26}$. Finalmente, a pesar de que este trabajo realiza una estimación con datos actualizados de la población española, sería interesante comprobar la precisión de nuestras estimaciones en registros poblacionales. 


\section{CONCLUSIONES}

En este trabajo se ha estimado que en España existen unos 17.100 casos prevalentes de ICC con valores de potasio plasmático $\geq 5,5 \mathrm{mmol} / \mathrm{L}$ (unos 10.000 hombres y unas 7.100 mujeres) y que se dan unos 14.900 casos nuevos anualmente (unos 9.500 hombres y unas 5.400 mujeres). Estas estimaciones ponen de manifiesto la necesidad de investigar nuevas estrategias terapéuticas fácilmente toleradas para controlar la hiperpotasemia en los pacientes de ICC por el gran número de individuos que podrían beneficiarse de medidas profilácticas y porque esta condición aumenta sensiblemente la morbimortalidad de estos sujetos.

\section{FINANCIACIÓN}

El presente trabajo ha sido financiado en parte por la Agencia de Gestión de Ayudas Universitarias y de Investigación (AGAUR) (2014-SGR-240), el CIBER de Fisiopatología de la Obesidad y Nutrición (CIBEROBN), el CIBER de Enfermedades Cardiovasculares (CIBERCV) y fondos FEDER de la Unión Europea.

\section{CONFLICTO DE INTERESES}

Ninguno. 


\section{BIBLIOGRAFÍA}

1. Sarwar CMS, Papadimitriou L, Pitt B, Piña I, Zannad F, Anker SD, et al. Hyperkalemia in Heart Failure. J Am Coll Cardiol. 2016;68(14):1575-89.

2. Khanagavi J, Gupta T, Aronow WS, Shah T, Garg J, Ahn C, et al. Hyperkalemia among hospitalized patients and association between duration of hyperkalemia and outcomes. Arch Med Sci. 2014;10(2):251-7.

3. Jain N, Kotla S, Little BB, Weideman RA, Brilakis ES, Reilly RF, et al. Predictors of hyperkalemia and death in patients with cardiac and renal disease. Am J Cardiol. 2012;109(10):1510-3.

4. An JN, Lee JP, Jeon HJ, Kim DH, Oh YK, Kim YS, et al. Severe hyperkalemia requiring hospitalization: predictors of mortality. Crit Care. 2012;16(6):R225.

5. McCullough PA, Beaver TM, Bennett-Guerrero E, Emmett M, Fonarow GC, Goyal A, et al. Acute and chronic cardiovascular effects of hyperkalemia: new insights into prevention and clinical management. Rev Cardiovasc Med. 2014;15(1):11-23.

6. Pitt B, Rossignol P. Potassium lowering agents: Recommendations for physician and patient education, treatment reappraisal, and serial monitoring of potassium in patients with chronic hyperkalemia. Pharmacol Res. 2017;118:2-4.

7. Rossignol P, Legrand M, Kosiborod M, Hollenberg SM, Peacock WF, Emmett M, et al. Emergency management of severe hyperkalemia: Guideline for best practice and opportunities for the future. Pharmacol Res. 2016;113(Pt A):58591.

8. Desai AS. Hyperkalemia in patients with heart failure: incidence, prevalence, and management. Curr Heart Fail Rep. 2009;6(4):272-80.

9. Crespo-Leiro MG, Segovia-Cubero J, González-Costello J, Bayes-Genis A, López-Fernández S, Roig E, et al. Adherence to the ESC Heart Failure Treatment Guidelines in Spain: ESC Heart Failure Long-term Registry. Rev Esp Cardiol (Engl Ed). 2015;68(9):785-93. 
10. Anguita-Sánchez M, Crespo-Leiro MG, de Teresa-Galván E, Jiménez-Navarro M, Alonso-Pulpón L, Muñiz-García J, et al. Prevalence of heart failure in the Spanish general population aged over 45 years. The PRICE Study. Rev Esp Cardiol. 2008;61(10):1041-9.

11. Sayago-Silva I, García-López F, Segovia-Cubero J. Epidemiología de la insuficiencia cardiaca en España en los últimos 20 años. Rev Esp Cardiol. 2013;66(8):649-56.

12. Anguita-Sánchez M, Ojeda-Pineda S. Diagnóstico y tratamiento de la insuficiencia cardíaca diastólica. Rev Esp Cardiol. 2004;57(6):570-5.

13. Mosterd A, Hoes AW, de Bruyne MC, Deckers JW, Linker DT, Hofman A, et al. Prevalence of heart failure and left ventricular dysfunction in the general population; The Rotterdam Study. Eur Heart J. 1999;20(6):447-55.

14. Davies M, Hobbs F, Davis R, Kenkre J, Roalfe AK, Hare R, et al. Prevalence of left-ventricular systolic dysfunction and heart failure in the Echocardiographic Heart of England Screening study: a population based study. Lancet. $2001 ; 358(9280): 439-44$.

15. Redfield MM, Jacobsen SJ, Burnett JC, Mahoney DW, Bailey KR, Rodeheffer RJ. Burden of systolic and diastolic ventricular dysfunction in the community: appreciating the scope of the heart failure epidemic. JAMA. 2003;289(2):194202.

16. Valle R, Baccichetto R, Barro S, Calderan A, Carbonieri E, Chinellato M, et al. Heart failure in Eastern Veneto: prevalence, hospitalization rate, adherence to guidelines and social costs. Monaldi Arch Chest Dis. 2006;66(1):63-74.

17. Tiller D, Russ M, Greiser $\mathrm{KH}$, Nuding S, Ebelt $\mathrm{H}$, Kluttig A, et al. Prevalence of Symptomatic Heart Failure with Reduced and with Normal Ejection Fraction in an Elderly General Population-The CARLA Study. PLoS One. 2013;8(3):e59225.

18. Schwarzer G. meta: An R package for meta-analysis. R News. 2007;7(3):40-5. 
19. Instituto Nacional de Estadística. Cifras de población residente en España. 2016. Available from:

http://www.ine.es/dyngs/INEbase/es/operacion.htm?c=Estadistica_C\&cid=12547 $36176951 \&$ menu $=u$ ultiDatos\&idp $=1254735572981$

20. Crespo-Leiro MG, Anker SD, Maggioni AP, Coats AJ, Filippatos G, Ruschitzka F, et al. European Society of Cardiology Heart Failure Long-Term Registry (ESC-HF-LT): 1-year follow-up outcomes and differences across regions. Eur $\mathrm{J}$ Heart Fail. 2016;18(6):613-25.

21. Hoss S, Elizur Y, Luria D, Keren A, Lotan C, Gotsman I. Serum Potassium Levels and Outcome in Patients With Chronic Heart Failure. Am J Cardiol. $2016 ; 118(12): 1868-74$.

22. Martín-Pérez M, Ruigómez A, Michel A, García Rodríguez LA. Impact of hyperkalaemia definition on incidence assessment: implications for epidemiological research based on a large cohort study in newly diagnosed heart failure patients in primary care. BMC Fam Pract. 2016;17(1):51.

23. Bakris GL, Pitt B, Weir MR, Freeman MW, Mayo MR, Garza D, et al. Effect of Patiromer on Serum Potassium Level in Patients With Hyperkalemia and Diabetic Kidney Disease. JAMA. 2015;314(2):151.

24. Pitt B, Anker SD, Bushinsky DA, Kitzman DW, Zannad F, Huang I-Z, et al. Evaluation of the efficacy and safety of RLY5016, a polymeric potassium binder, in a double-blind, placebo-controlled study in patients with chronic heart failure (the PEARL-HF) trial. Eur Heart J. 2011;32(7):820-8.

25. US Food \& Drug Administration. The Drug Development Process - Step 3:

Clinical Research. Available from:

https://www.fda.gov/forpatients/approvals/drugs/ucm405622.htm

26. Muzzarelli S, Maeder MT, Toggweiler S, Rickli H, Nietlispach F, Julius B, et al. Frequency and Predictors of Hyperkalemia in Patients $\geq 60$ Years of Age With Heart Failure Undergoing Intense Medical Therapy. Am J Cardiol. 
2012;109(5):693-8.

27. Raymond I, Pedersen F, Steensgaard-Hansen F, Green A, Busch-Sorensen M, Tuxen C, et al. Prevalence of impaired left ventricular systolic function and heart failure in a middle aged and elderly urban population segment of Copenhagen. Heart. 2003;89(12):1422-9. 


\section{TABLAS}

Tabla 1. Estimación del número de pacientes con hiperpotasemia $\left(\mathrm{K}^{+} \geq 5,5 \mathrm{mmol} / \mathrm{L}\right) \mathrm{e}$ insuficiencia cardiaca crónica (fracción de eyección ventricular $\leq 40 \%$ ) en España (por grupos de edad y sexo).

\begin{tabular}{|c|c|c|c|c|c|}
\hline & & Prevalencia & $N$ pacientes & Prevalencia & $\begin{array}{r}N \text { pacientes } \\
\text { ICC-HK }\end{array}$ \\
\hline \multicolumn{6}{|l|}{ Hombres } \\
\hline 45-54 años & 3.599 .237 & 0,0058 & 20.876 & 0,0337 & 704 \\
\hline 55-64 años & 2.762 .924 & 0,0217 & 59.955 & 0,0337 & 2.020 \\
\hline 65-74 años & 2.032 .554 & 0,0448 & 91.058 & 0,0337 & 3.069 \\
\hline 75 o más años & 1.720 .925 & 0,0720 & 123.907 & 0,0337 & 4.176 \\
\hline Total & & & & & 9.969 \\
\hline \multicolumn{6}{|l|}{ Mujeres } \\
\hline 45-54 años & 3.573 .742 & 0,0009 & 3.216 & 0,0337 & 108 \\
\hline 55-64 años & 2.879 .227 & 0,0052 & 14.972 & 0,0337 & 505 \\
\hline $65-74$ años & 2.291 .671 & 0,0178 & 40.792 & 0,0337 & 1.375 \\
\hline 75 o más años & 2.656 .231 & 0,0578 & 153.530 & 0,0337 & 5.174 \\
\hline Total & & & & & 7.162 \\
\hline \multicolumn{3}{|c|}{ Hombres y mujeres, total } & & & 17.131 \\
\hline
\end{tabular}

HK: hiperpotasemia; ICC: insuficiencia cardiaca crónica. 
Tabla 2. Estimación del número de pacientes con insuficiencia cardiaca crónica (fracción de eyección ventricular $\leq 40 \%)$ que desarrollarán hiperpotasemia $\left(K^{+} \geq 5,5\right.$ $\mathrm{mmol} / \mathrm{L}$ ) cada año en España (por grupos de edad y sexo).

\begin{tabular}{|c|c|c|c|c|c|}
\hline Sexo y edad & $N(2016)$ & $\begin{array}{r}\text { Prevalencia } \\
\text { ICC }\end{array}$ & $\begin{array}{r}N \text { pacientes } \\
\text { ICC }\end{array}$ & $\begin{array}{l}\text { Incidencia } \\
\text { HK en ICC }\end{array}$ & $\begin{array}{r}N \text { nuevos } \\
\text { casos ICC-HK }\end{array}$ \\
\hline \multicolumn{6}{|l|}{ Hombres } \\
\hline 45-54 años & 3.599 .237 & 0,0058 & 20.876 & $\begin{array}{l}1,97 \text { (45-49); } \\
2,21(50-54) \\
2,21(55-59)\end{array}$ & 435 \\
\hline 55-64 años & 2.762 .924 & 0,0217 & 59.955 & $\begin{array}{l}3,16(60-64) \\
3,16(65-69)\end{array}$ & 1.582 \\
\hline 65-74 años & 2.032 .554 & 0,0448 & 91.058 & $\begin{array}{l}3,58(70-74) \\
3,58(75-79):\end{array}$ & 3.050 \\
\hline $\begin{array}{l}75 \text { o más años } \\
\text { Total }\end{array}$ & 1.720 .925 & 0,0720 & 123.907 & $3,62(80+)$ & $\begin{array}{l}4.466 \\
9.533\end{array}$ \\
\hline \multicolumn{6}{|l|}{ Mujeres } \\
\hline 45-54 años & 3.573 .742 & 0,0009 & 3.216 & $\begin{array}{l}1,43(45-49) \\
1,61(50-54) \\
1,61(55-59)\end{array}$ & 49 \\
\hline 55-64 años & 2.879 .227 & 0,0052 & 14.972 & $\begin{array}{l}2,30(60-64) \\
2,30(60-64)\end{array}$ & 289 \\
\hline 65-74 años & 2.291 .671 & 0,0178 & 40.792 & $\begin{array}{l}2,60(65-69) \\
2,60(70-74) ;\end{array}$ & 995 \\
\hline 75 o más años & 2.656 .231 & 0,0578 & 153.530 & $2,63(80+)$ & 4.023 \\
\hline Total & & & & & 5.356 \\
\hline Hombres y muj & s, total & & & & 14.889 \\
\hline
\end{tabular}

HK: hiperpotasemia; ICC: insuficiencia cardiaca crónica. 
FIGURAS

FIGURA 1. Meta-análisis de la prevalencia de insuficiencia cardiaca crónica (fracción de eyección ventricular $\leq 40 \%$ ) en población general europea/norteamericana en hombres en diferentes franjas de edad.

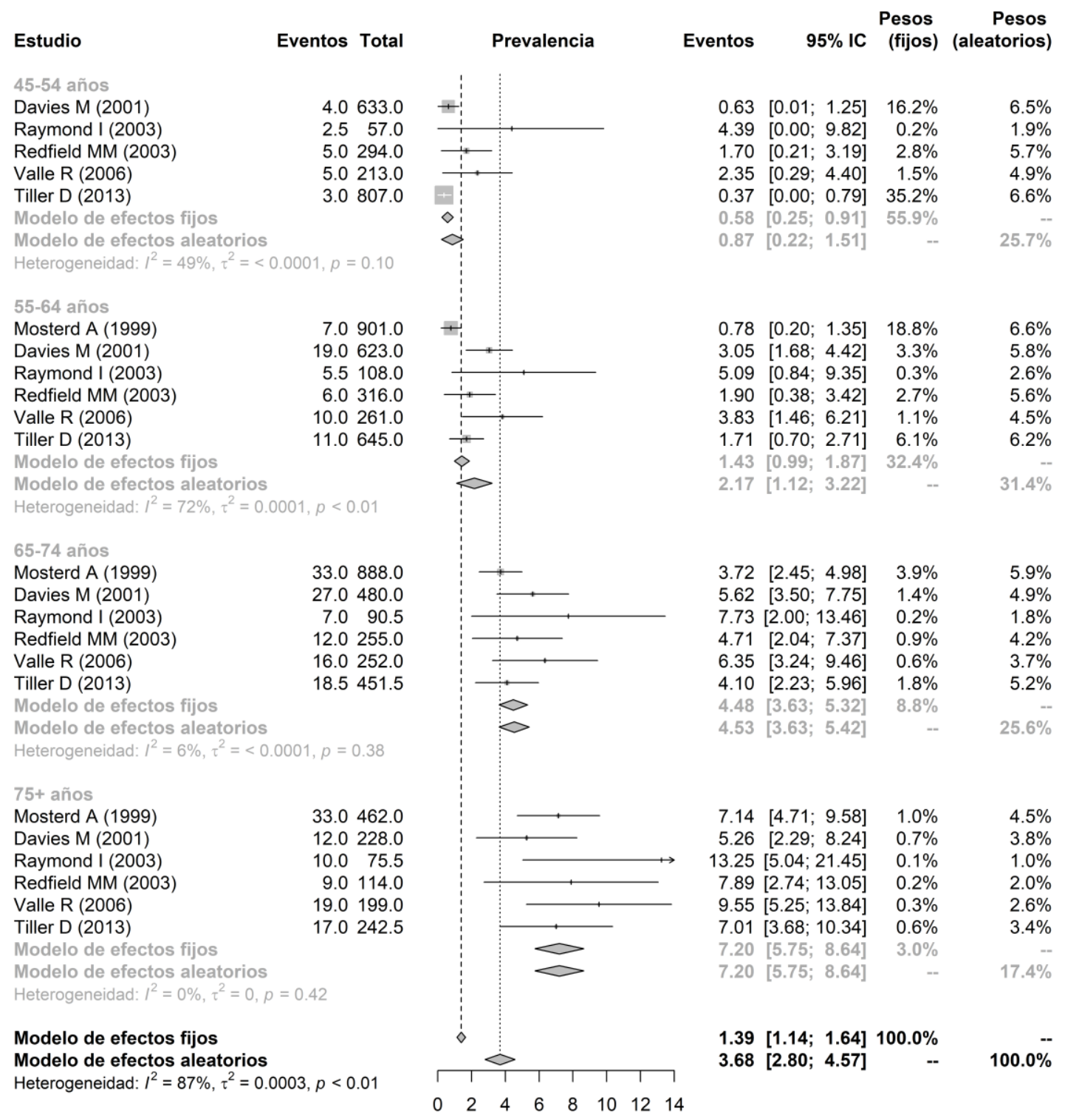


FIGURA 2. Meta-análisis de la prevalencia de insuficiencia cardiaca crónica (fracción de eyección ventricular $\leq 40 \%$ ) en población general europea/norteamericana en mujeres en diferentes franjas de edad.

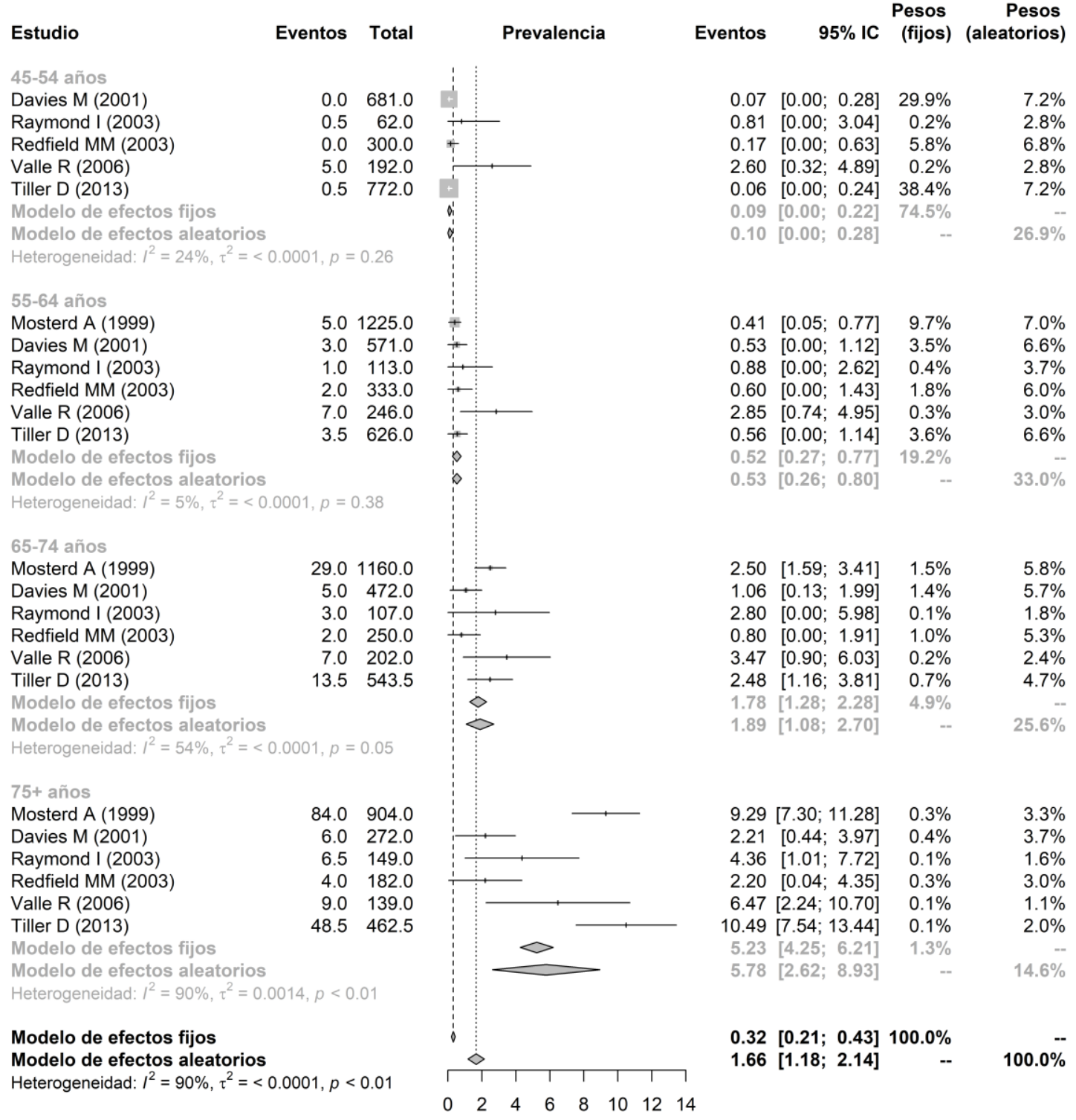


FIGURA 3. Meta-análisis de la prevalencia de hiperpotasemia $\left(\mathrm{K}^{+} \geq 5,5 \mathrm{mmol} / \mathrm{L}\right)$ en estudios con pacientes con insuficiencia cardiaca crónica.

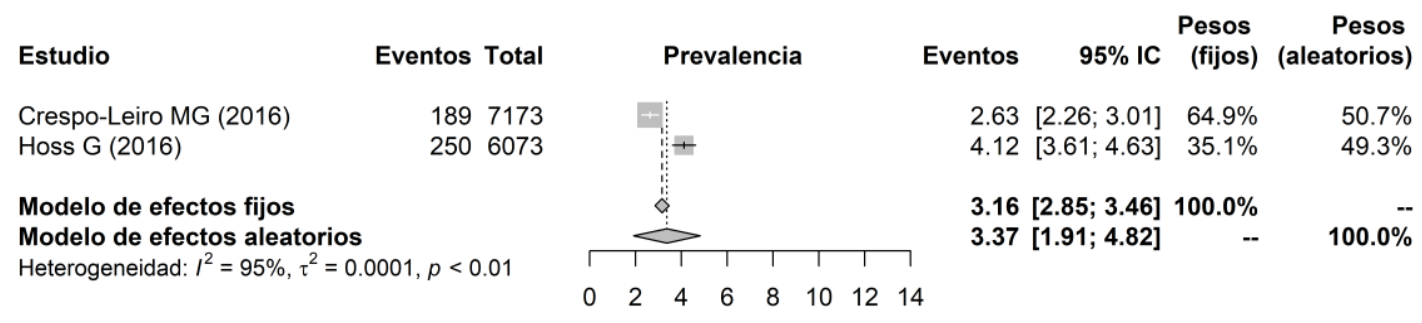

